\title{
CREATIVE THINKING AND RANDOM NUMBER GENERATION TEST
}

\author{
KIMIHIKO MATSUDA ${ }^{1,2}$ \\ Department of Psychology, Kyushu Ciniversity
}

\begin{abstract}
In S.I. model (Structure of Intelligence), Guilford (1971) referred the concept of divergent production as one of the most important factors concerning creative thinking. On the other hand, Murakami (1972) has been developing Random Number Generation Test in order to measure the ability of human information processing and he introduced the concept of probablistic information processing for this purpose. In this study, the author intended to demonstrate the following 2 points; (a) there was a significant correlation between these two conceptsdivergent production suggested by Guilford and probablistic information processing suggested by Murakami, (b) Random Number Generation Test was one of the desirable tests concerning DS factor (divergent production of symbolic material). These 2 points were approximately clarified.
\end{abstract}

It is often believed that if a person shows strong rigidity of behavior in one area he will be likely to show it in many others, including sensory or perceptual activities, thinking, attitudes and so on. Such a hypothesis would call for substantial correlation among various kinds of indices of rigidity vs. Hexibility in all the areas of behavior. But according to recent reports concerning rigidity of behavior, one can hardly fail to obtain the following impression. There is not one general trait of rigidity that is common to all kinds of behaviors-sensory, perceptual, thinking, psychomotor, and attitudinal, rather there are several more limited traits of rigidity (or of flexibility) which are specific to each area. Thurstone (1944) reported a factor that he described as flexibility in dealing with perceptual organization, or a freedom from gestaltbindung. In several studies it has been found that there appears to be a rigidity factor common to certain psychomotor tests studied by Cattell and

1 The author is indebted to Mr. Murakami for drawing his attention to this problem.

${ }_{2}^{2}$ Requests for reprints should be sent to Kimihiko Matsuda, Department of psychology, Kyushu University, Hakozaki, Higashi-ku, Fukuoka 812, Japan.
Tiner (1949).

In 1954, Guilford and his associates began to study creative thinking abilities and found that there were two distinct flexibility factors in the area of thinking which they designated as spontaneous flexibility and adaptive flexibility (1954). Further, they found that spontaneous flexibility and adaptive flexibility were qualities psychologically opposite to two kinds of rigidity of thinking-perseveration and persistencerespectively (Frick, Guilford, Christensen, \& Merrifield 1959). Guilford (1971) presented a S.I. model (structure of intelligence), and classified spontaneous flexibility into DM factor (divergent production of semantic material) and adaptive flexibility into DF factor (divergent production of figural material), and said that such a divergent thinking pattern was one of the most important factors concerning creative thinking.

On the other hand, Kimikatsu Murakami (1972), had been developing the Random Number Generation Test (R.N. G.T.) to investigate the abilities and characteristics of human information processing. According to his theory, this R.N.G.T. reflects the structural differences of individual information processing and they can be 
evaluated in terms of speed and flexibility of the conceptual association. R.N.G.T. was based on the following assumption: although a variety of transformation done in the course of human information processing (for example, composition, decomposition, or organization of concepts) depends on the direction of processing and the contents of stored memory, its processing pattern can be regarded as a simple process of transition in which a concept changes from one to another successively. Here two types of transition can be considered: one in which the path of transition is determined beforehand and there is no room for free choice, and another in which the path of transition is not predetermined and all choices of the paths are free. If the concept of Markov multi-decision processes is applied to these typical types of processing, the former can be regarded as a deterministic processing whose path of transition depends wholly on one's memory, and the latter as a probablistic processing. Although a practical pattern of human information processing is composed of a mixture of these two extreem types, it seems that the essential characteristics of individual information processing appear in the latter case. If the practical case of human information processing is taken into consideration, Murakami's assumption seems to have some doubtful points, but his conclusion that probablistic information processing is one of the clues by which the ability of creative information processing can be clarified and his intention to use R.N.G.T. for this purpose are acceptable.

It seems that the concept of probablistic information processing suggested by Murakami corresponds to the concept of divergent production suggested by Guilford as an important factor concerning creative thinking, and therefore, the main purpose of this study is to investigate the relation between these two concepts.

\section{STUDY I}

The purpose of this study I is to introduce the tests which investigate the two concepts mentioned above and to provide basic data for further study.

Usually, divergent production is evaluated from two points of view-qualitative and quantitative, and therefore, Guilford (1959, 1971) referred to the quantitative side as fluency and to the qualitative side as flexibility in his study.

\section{Method}

Tests. 1. The test of divergent production

In this study, spontaneous flexibility was used as a representation of the concept of divergent production, and Word Association Test (W.A. T.) was used as an index of spontaneous flexibility. The main reason W.A.T. was used as a test of divergent production was that this could provide both quantitative and qualitative data at the same time. In this W.A.T., subjects were asked to associate 4 words with one stimulus word within $30 \mathrm{sec}$. The test was composed of 18 stimulus words chosen from the Association Frequency Table (Umemoto, 1969), and though the stimulus words were nouns, verbs, and adjectives, the response words were not limited at all regarding parts of speech.

The qualitative side of the response was measured by the following procedure. First, each response word was classified into three groups on the basis of its value of association (the value of association was determined according to Umemoto's Association Frequency Table) and given a score in relation to its value. This score expressed the strength of perseveration in association; in other words, a response given a high score meant an ordinary and commonplace association. Next, the average score of all response words of each subject was calculated and it was regarded as his perseveration score. The reason for the use of the average score as an index of the individual perseveration score was that the number of response words was different among individuals ; accordingly, 
the total score of each word could not be a correct index.

With regard to the quantitative side of the response, the total number of response words was used as its index.

The reliability of this W.A.T. was estimated by the split-half technique; on the qualitative side it was .72 , and on the quantitative side it was .75 .

2. The test of probablistic information processing

R.N.G.T. was used, based on the following deduction. Human is naturally used to treating numbers in a logical way, and not accustomed to thinking in such a way as to arrange numbers randomly. Therefore, the random numbers created by human whose thinking and behavior are bound by the low of causation cannot be regarded as random numbers in the strict sense of the word where there is no correlation between adjacent numbers; but on the other hand, since each person exerts conscious control in his own way, considerable differences among individuals can be observed. Accordingly, it is expected that R.N.G.T. reflects the differences among individuals in the mode of thinking.

The following is the procedure used in R.N. G.T. and the method of analyzing data. Subjects were given a blank paper and asked to write one-digit numbers (0-9), maintaining the following two conditions at the same time and the same degree.

(a) Don't arrange numbers regularly.... (qualitative condition)

(b) Write as many numbers as you can.... (quantitative condition)

The test consisted of 4 trials, and the time limit for a trial was one minute. The first three trials were repeated under the same conditions [maintaining both (a) and (b)] at intervals of $30 \mathrm{sec}$. On the forth trial, subjects were asked to arrange one-digit numbers in increasing order repeatedly, maintaining only (b) condition.

Though Murakami reported several methods of analyzing the data, calculating time (C.T.) and degree of randomness (R.D.) were mainly used in this study. First, let's explain C.T..
This was used as a quantitative score of R.N.G. T. and C.T. in the $i$ th trial, (C.T. $)_{i}$, was given by using the following equation

$$
(\text { (.T. })_{i}=60 /(\mathrm{G} . \mathrm{N} .)_{i}-60 / \text { R.N. }
$$

and C.T. through the first three trials was given as follows :

$$
(\mathrm{C} . \mathrm{T} .)=180 /(\mathrm{G} . \mathrm{N} .)_{t}-60 / \mathrm{R} . \mathrm{N} \text {. }
$$

[(G.N. $)_{i}$ : the number of figures generated in the $i$ th trial; (G.N.) $)_{t}$ the total number of figures generated in the first three trials under the same condition; R.N.: the number of figures generated in the fourth trial]. R.D. was used as an index of the qualitative side of R.N.G.T.. Generally, there is a remarkable deviation in the transition width (Wk) of random numbers created by human.

$$
W \mathrm{k}=\gamma_{\mathrm{k}+1}-\gamma_{\mathrm{k}}
$$

here, $\gamma_{k}$ represents the figure generated $k$ th in a trial. Therefore, R.D. was defined as follows in order to investigate such random numbers.

$$
\text { R.D. = F.D.D. + T.W.D. }
$$

F.D.D. represents the deviation in frequency distribution and was given by the following equation

$$
\text { F.D.D. }=\sum_{l=0}^{9}|n l| N-0.1 \mid
$$

( $n l$ : the number of figure $l$ generated in a trial ; $N$ : the number of figures generated in a trial). T.W.D. represents the probable deviation in transition width and was given by the following equation

$$
\text { T.W.D }=\sum_{i=-9}^{9}\left|\mathrm{P}_{i}-(\mathrm{Pr})_{i}\right|
$$

here

$$
\begin{aligned}
& \mathrm{P}_{i}=\frac{n_{i}}{\mathrm{~N}-1}=\frac{\sum_{\mathrm{k}=1}^{\mathrm{N}-1} \delta \mathrm{Wk} \cdot i}{\sum_{i=-9}^{9} n_{i}} \\
& (\mathrm{Pr})_{i}=\frac{(10-|i|)}{100}
\end{aligned}
$$

[ $n i$ : frequency in the case where $W \mathrm{k}$ is equal to $i ; i=0, \pm 1, \pm 2, \ldots . ., \pm 9 ; \mathbf{P}_{i}$ : probable transition width; $(\operatorname{Pr})_{i}$ : probable transition 
TABLE la

Transition matrixt, $\uparrow$

\begin{tabular}{l|l|l|l|l|l|l|l|l|l|l|}
\hline & 0 & 1 & 2 & 3 & 4 & 5 & 6 & 7 & 8 & 9 \\
\hline 0 & & 1 & 2 & 1 & 1 & & & & 2 & 1 \\
\hline & 3 & & 1 & 1 & & & 1 & & & \\
\hline 2 & 2 & 3 & 1 & & 3 & & & 2 & 3 & 1 \\
\hline 3 & 2 & & 2 & & & 1 & 2 & & 1 & \\
\hline 4 & & & & 1 & & 4 & & & 2 & \\
\hline 5 & & & 1 & & 1 & & 2 & & 1 & 1 \\
\hline 6 & 1 & & 4 & 2 & & & 1 & 2 & 1 & 1 \\
\hline 7 & & 1 & 3 & 2 & & 1 & & 1 & 1 \\
\hline 8 & & & 1 & & 1 & & 3 & 5 & & 1 \\
\hline 9 & & 1 & 2 & & & 1 & 2 & & & \\
\hline
\end{tabular}

t The number of figures generated in this trial is 89 .

t† T. W. D. between adjacent numbers is .466 .

width in a sequence of random numbers which fill cells of a transition matrix (Table 1) with the same number uniformly]. These two indices (C.T., R.D.) show that the lower their values are, the better the results of the test are.

Subjects. 67 freshmen at a women's university were used as subjects, and the range of their ages was from 18 to 19 .

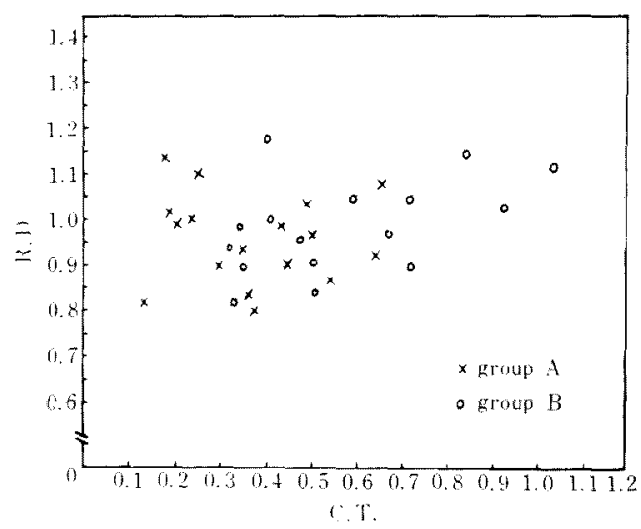

FIG. 1. Qualitative side and quantitive side in R. N. G. T
TABLE 1b

Transition matrixt, $+\uparrow$

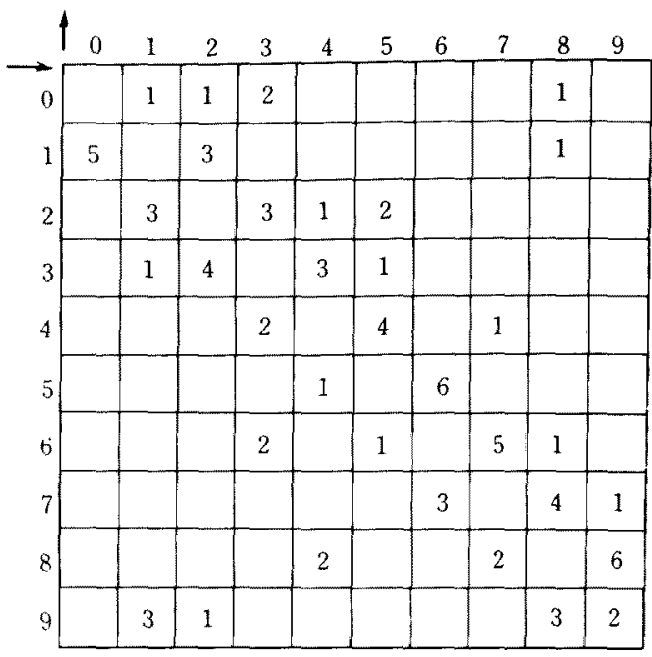

$t$ The number of figures generated in this trial is 83 .

†† T.W. D. between adjacent number is 1.106 .

\section{Results and Consideration}

Quantitative side. On the basis of the results of W.A.T., the 67 subjects were classified into two groups, group $\mathrm{A}$ and group B. Subjects who had a large total number of response words were assigned to group A (17 subjects), and those who had smaller total number of response words were assigned to group B (16 subjects), and these two groups were compared in regard to C.T. and R.D. (Fig. 1). In Fig. 1, the horizontal scale shows quantitative side and the vertical scale shows qualitative side; therefore, the position of a point plotted in Fig. 1 is determined by both C.T. and R.D. Though these two groups did not differ statistically in R.D. (and there were few differences in both F.D.D. and T.W.D.), they differed significantly in C.T. $(t=2.11, d f=31$, $p<0.5$ ). In other words, subjects who showed good results quantitatively in W.A. $T$. also showed good results quantitatively in R.N.G.T., but qualitatively there was no relation between W.A.T. and R.N.G. 
TABLE 2

Comparative table between group $\mathrm{C}$ and group D

\begin{tabular}{|c|c|c|c|c|c|c|c|c|}
\hline \multirow{2}{*}{ group } & \multicolumn{2}{|c|}{ F.D.D. } & \multicolumn{2}{|c|}{ T.W.D. } & \multicolumn{2}{|c|}{ R.D. } & \multicolumn{2}{|c|}{ C.T. } \\
\hline & C & D & C & D & $\mathrm{C}$ & D & C & $\mathrm{D}$ \\
\hline & .339 & .231 & .743 & .683 & 1.082 & .914 & .299 & .351 \\
\hline & .300 & .372 & .651 & .652 & .951 & 1.024 & .583 & .666 \\
\hline & .363 & .291 & .837 & .567 & 1.200 & .858 & .649 & .554 \\
\hline & .319 & .259 & .658 & .667 & .977 & .926 & .251 & .308 \\
\hline & .408 & .274 & .743 & .607 & 1.151 & .881 & .234 & .615 \\
\hline & .223 & .190 & .690 & .591 & .913 & .781 & .613 & .253 \\
\hline & .221 & .230 & .671 & .579 & .892 & .809 & .198 & .359 \\
\hline & .283 & .254 & .578 & .569 & .861 & .823 & .388 & .132 \\
\hline & .331 & .205 & .658 & .549 & .989 & .754 & .536 & .182 \\
\hline & .231 & & .631 & & .862 & & .444 & \\
\hline & .242 & & .546 & & .788 & & .450 & \\
\hline $\mathrm{m}$ & .307 & .285 & .671 & .611 & .978 & .897 & .407 & .355 \\
\hline s & .072 & .077 & .079 & .055 & .124 & .081 & .138 & .170 \\
\hline$t$ & \multicolumn{2}{|c|}{0.705} & \multicolumn{2}{|c|}{$2.07 *$} & \multicolumn{2}{|c|}{1.77} & \multicolumn{2}{|c|}{0.79} \\
\hline
\end{tabular}

T. . The coefficient of correlation between C.T. and the total number of response words in W.A.T. was $.39(t=2.30$, $d f=31, p<.05$ ), and this supported the fact mentioned above. The reliability of C.T. was estimated by a sort of internal consistency technique, and was .87. [Although C.T. as an index of each subject was calculated by equation (2), it was the average of three C.T. scores obtained by equation ' ( 1 ). Correlation among these three C.T. scores shows the degree of internal consistency, and therefore, the average value of these correlation coefficients was used as the reliability of C.T.].

Qualitative side. In analyzing the results of R.N.G.T., the following two groups were compared. One of them consisted of 14 subjects who obtained a high perseveration score in W.A.T. (group C), and the other consisted of 11 subjects who obtained a low perseveration score in W.A. T. (group D). Table 2 is a comparative table. It could be accepted that these two groups did not differ significantly in C.T., but it was unexpected that they did not show a significant difference in R.D. as well. If we examine R.D. in detail, it can be found that although there was no significant difference in F.D.D., there was a significant difference in T.W.D. $(t=2.07$, $d f=24, p<.05)$. Therefore, it can be said that T.W.D. is more closely related to the perseveration score than F.D.D.; that is to say, the coefficient of correlation between T.W.D. and the perseveration score was $.38(t=2.22, d f=24, p<.05)$, but the coefficient of correlation between F.D.D. and the perseveration score was $.18(t=$ $1.19, d f=24$, n.s.). The coefficient of correlation between T.W.D. and F.D.D. was .21 , and not statistically significant. Generally, we could observe only slight differences among individuals in the case of F.D.D., and the results obtained here in regard to F.D.D. agreed with the report by Murakami (1972).

In general, the results described so far agreed approximately with those expected; that is, we could show the expected relationship between the concept of spontaneous flexibility suggested by 
Guilford and the concept of probablistic information processing suggested by Murakami. But this relationship, as we observed it, was weaker than we had expected. Though several factors that might have caused this effect can be listed up, perhaps the difference in the kinds of material used in each test could be regarded as one of the main causes (the material of W.A.T. is a semantic one and the material of R.N. G.T. is a symbolic one). However, in the case of T.W.D. it seems that there is some room for improvement of analytic method, and if this can be improved, it will be possible to obtain a higher correlation than we did between these two concepts. For the present, we can not think of better way of expressing the quantitative side of R.N. G.T. using C.T. as its index, but we have reservation about the quantitative side. Though R.N.G.T. has a weak relationship with W.A.T. qualitatively if T.W.D. is used as a qualitative index of R.N.G.T., T.W.D. values of the first three trials showed a considerable deviation. Accordingly, the reliability of T.W.D. was not so high $(r=.48)$. The reliability of T.W.D. was estimated in the same way as C.T. .

\section{STUdy II}

The purpose of this study was to improve the analytic method in order to increase the reliability of T.W.D. and to investigate the relationship between two concepts more exactly. As long as we depend only on the analytic method described in study
I, we cannot learn anything about structural characteristics of random numbers created by human.

\section{Method}

Some aspects of structural characteristics can be shown by using a graph of the transition rhythm where the vertical scale shows the distance between adjacent numbers and the horizontal scale shows the arrangement order of random numbers (Fig. 2). If we investigate Fig. 2, it will be noticed that random numbers created by humans form many small and similar groups which contain about 4 or 5 numbers, and a graph of a transition rhythm exhibits many similar patterns which are repeated periodically. But when T.W.D. is calculated according to Murakami's equation it does not express these characteristics, and the result of R.N.G.T. cannot reflect the characteristics of human thinking sufficiently. As Murakami devised the R.N.G.T. on the basis of the Markov process, it is understandable that he considered T.W.D. between only adjacent numbers, but in order to express the characteristics mentioned above, it is necessary to investigate T.W.D. not only adjacent numbers but also alternate numbers or every three or every four numbers. If we want to obtain T.W.D. between alternate numbers, we have only to change the equations (3) and (7) as follows :

$$
\begin{aligned}
& \mathrm{Wk}=\gamma_{\mathrm{k}+2}-\gamma_{\mathrm{k}} \\
& \mathrm{P}_{i}=\begin{array}{c}
n_{i} \\
\mathrm{~N}-2
\end{array}=\frac{\sum_{\mathrm{k}=1}^{\mathrm{N}-2} \delta \mathrm{Wk} \cdot i}{\sum_{i=-9}^{9}}
\end{aligned}
$$

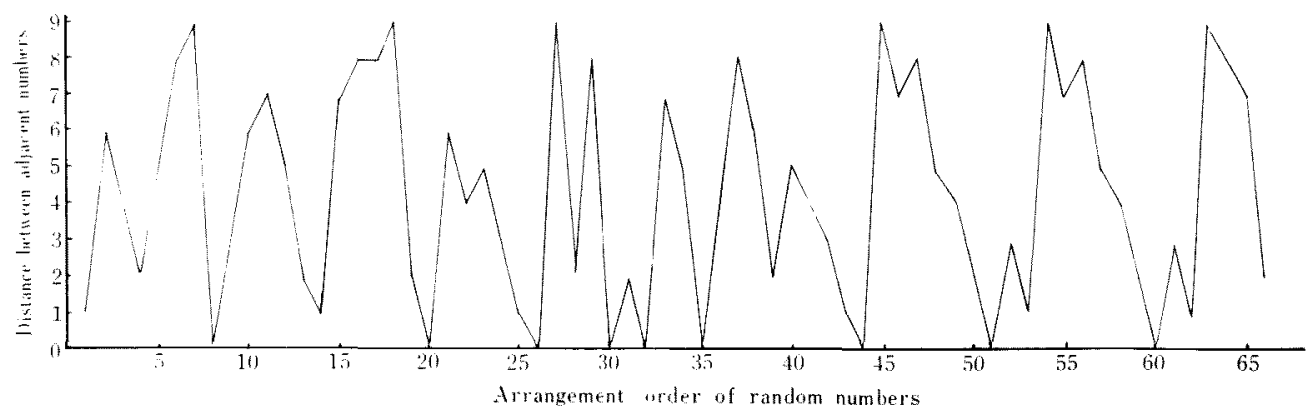

Fig. 2. A graph of the transition rhythm. 
Table 3

Four kinds of T.W.D, value for three groups of subjects

\begin{tabular}{|c|c|c|c|c|c|c|c|c|c|c|c|c|c|c|c|}
\hline \multirow{2}{*}{$\begin{array}{l}\text { T.W.D. } \\
\text { group }\end{array}$} & \multicolumn{3}{|c|}{$\begin{array}{l}\text { between } \\
\text { adjacent numbers }\end{array}$} & \multicolumn{3}{|c|}{$\begin{array}{l}\text { between } \\
\text { alternate } \\
\text { numbers }\end{array}$} & \multicolumn{3}{|c|}{$\begin{array}{l}\text { between every } \\
\text { three numbers }\end{array}$} & \multicolumn{3}{|c|}{$\begin{array}{l}\text { between every } \\
\text { four numbers }\end{array}$} & \multicolumn{3}{|c|}{$\begin{array}{c}\text { average value } \\
\text { of four kinds } \\
\text { of T.W.D. }\end{array}$} \\
\hline & C & D & $\mathbf{E}$ & C & $\mathrm{D}$ & $\mathrm{E}$ & $\mathrm{C}$ & $\mathrm{D}$ & $\mathrm{E}$ & C & $\mathrm{D}$ & $\mathrm{E}$ & C & $\mathrm{D}$ & $\mathrm{E}$ \\
\hline & .743 & .683 & .805 & .561 & .518 & .709 & .502 & .375 & .670 & .538 & .392 & .521 & .586 & .492 & .672 \\
\hline & .651 & .652 & .846 & .593 & .514 & .726 & .549 & .492 & .680 & .420 & .477 & .585 & .553 & .534 & .702 \\
\hline & .837 & .567 & .793 & .654 & 412 & .587 & .533 & .463 & .509 & .519 & .402 & 452 & .636 & .461 & .585 \\
\hline & .750 & .567 & .752 & .502 & .363 & .580 & .464 & .331 & .600 & .413 & .324 & .472 & .532 & .396 & .601 \\
\hline & .704 & .695 & .863 & .578 & .493 & .626 & .492 & .458 & .455 & .402 & .369 & .449 & .544 & .511 & .598 \\
\hline & .658 & .667 & .694 & .583 & .467 & .535 & .463 & .383 & .628 & .451 & .401 & .514 & .539 & .480 & .593 \\
\hline & $.7+3$ & .607 & .688 & .543 & .349 & .492 & .474 & .353 & .405 & .401 & .368 & .439 & .540 & .419 & .506 \\
\hline & .690 & .591 & .714 & .568 & .534 & .469 & .510 & .384 & .494 & .424 & .360 & .546 & .548 & .467 & .556 \\
\hline & .671 & .579 & .653 & .536 & .529 & .555 & .516 & .489 & .408 & .512 & .383 & .413 & .559 & .495 & .507 \\
\hline & .578 & .569 & .607 & .451 & .487 & .443 & .402 & .444 & .450 & .377 & .429 & .514 & .452 & .482 & .504 \\
\hline & .658 & .549 & .599 & .526 & .321 & .424 & .455 & .391 & .573 & .478 & .383 & .590 & .529 & .411 & .547 \\
\hline & .631 & & 621 & .467 & & .539 & .357 & & .468 & .388 & & .558 & .461 & & .567 \\
\hline & .546 & & & .442 & & & .363 & & & .360 & & & .428 & & \\
\hline & .530 & & & .438 & & & .496 & & & .449 & & & .478 & & \\
\hline $\mathrm{m}$ & .671 & .611 & .720 & .532 & .453 & .556 & .470 & .415 & .528 & .438 & .392 & .504 & .528 & .468 & .578 \\
\hline$s$ & .079 & .055 & .084 & .057 & .076 & .094 & .054 & .051 & .096 & .053 & .037 & .059 & .045 & .045 & .063 \\
\hline$F$ & & $6.08^{*}$ & & & $5.24 *$ & & & $90 * *$ & & & $65^{* *}$ & & & $1.7 * *$ & \\
\hline
\end{tabular}

T.W.D. between every three numbers or every four numbers is obtained by changing the equation (3) and (7) respectively as follows:

$$
\begin{aligned}
& W \mathrm{k}=i k+3-i \mathrm{k} \\
& \mathrm{P}_{i}=\frac{n_{i}}{v-3}=\frac{\sum_{\mathrm{k}=1}^{N-3} \delta \mathrm{Wk} \cdot i}{\sum_{i=-9}^{9} n_{i}} \\
& W \mathrm{k}=\gamma_{\mathrm{k}+4}-\gamma_{\mathrm{k}} \\
& \mathrm{P}_{i}=\frac{n_{i}}{N-4}=\sum_{k=1}^{N-4} \delta \mathrm{Wk} \cdot i \\
& \sum_{i=-9}^{9} n_{i}
\end{aligned}
$$

Wk expressed by (9), (11) and (13) means the transition width of the two-step, three-step, and four-step connections. In this study, we did not investigate more than a five-step connection, because most of the similar patterns that appeared in Fig. 2 were composed of 4 or 5 numbers.
Though these new analytic methods were applied to the same data as in study $I$, in study II the data obtained from twelve schizophrenic patients were added in order to examine construct validity of R.N.G.T.. The reason for choosing schizophrenic patients as subjects for this study was that a great deal of previous experimental researches had shown the existence of a strong perseveration tendency in their mode of thinking (for example, Kretschmer, 1955). Patients with slight symptoms were used as subjects, and their ages ranged 18 to 27.

\section{Results and consideration}

First of all, let's investigate to what degree the reliability of T.W.D. increased by using the new analytic method. As we looked in study I, the coefficient of reliability of T.W.D. between adjacent numbers was .48 , but the coefficient of relia- 
bility of T.W.D. between alternate numbers, every three numbers, and every four numbers were respectively $.51, .65$, and .59 ; furthermore, if the average value of these four T.W.D. was used as an index, the coefficient of reliability of T.W.D. was .69. As the reliability of T.W.D. increased, the correlation between T.W.D. and the subject's perseveration score on the W.A.T. became higher. For example, when T.W. $D$. between adjacent numbers was used as an index, the coefficient of correlation was .38 , but when an average value of these four T.W.D. was used as an index, it was $.52(t=2.51, p<.02)$.

Table 3 shows the four kinds of T.W.D. values for three groups of subjects. The member of group C and group D are the same as in Table 1, and group E consist of 12 schizophrenic patients. In the case of all four T.W.D., these three groups showed significant differences. This means that these three groups differ in probablistic information processing ability. As expected, group $\mathrm{E}$ showed the highest T.W.D. value of the three groups, group C the second highest T.W.D. value, and group D showed the lowest value of the three groups in all kinds of T.W.D.

From these results, it can be concluded that spontaneous flexibility and probablistic information processing ability affect the problem of divergent production from different angles, that is, the former is concerned with semantic material and the latter with symbolic material. If we classify them according to the S.I. model, the former is the DM factor and the latter is the DS factor, and R.N.G.T. is a valid index of the DS factor. Furthermore, R.N.G.T. can provide two kinds of dataquantitative and qualitative-at the same time, and in this sense R.N.G.T. can be said to be an ideal test of divergent production.

\section{REFERENCES}

Cattell, R. B., \& Tiner, L. G. 1949 The varieties of structural rigidity. Journal of personality, 17, 321-34l.

Frick, J. W., \& Gullford, J. P., Christensen, P. R., \& Merrifield, P. R. 1959 A factoranalytic study of Alexibility in thinking. Educational and psychological measurement, 19, 469 496.

Guilford, J. P., \& Hoephner, R. 1971 The analysis of intelligence. New York: McGrawHill.

Kretschmer, E. 1955 Körperbau und Charakter. 21/22 Aufl. Springer.

Murakami, K. 1972 Random number generation testing method. In Mori, M. (Ed.), Flexible thinking. 1-18, Tokyo: Jiji Press (Written in Japanese).

Thurstone, L. L. 1944 A factorical study of perception. Psychometric monographs, No. 4.

Uмемото, T. 1969 Association frequency table. Tokyo: University of Tokyo Press. (Written in Japanese).

Wilson, R. C., Gullford, J. P., Christensen. P. R., \& Lewis, D. J. 1954 A factor-analytic study of creative-thinking abilities. Psychometrika, 19, 297-311.

(Received March 15, 1973) 